\title{
Application of AI Technology in Patrol Inspection for Surface Environment of Transmission Channel
}

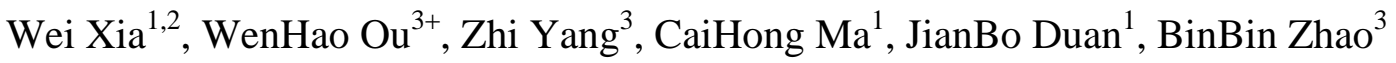 \\ ${ }^{1}$ Institute of Remote Sensing and Digital Earth, Chinese Academy of Sciences, Beijing, China \\ ${ }^{2}$ University of Chinese Academy of Sciences, Beijing, China \\ ${ }^{3}$ China Electric Power Research Institute Co., Ltd. , Beijing, China
}

\begin{abstract}
The artificial intelligence (AI) technology is one of the most subversive computer technologies. At present, the AI technology may also lead to better management and development of power industry, will advance the automation technologies of power system in a safety, reliable and highly-efficient direction, and enjoys bright development prospect in operation of power system, power transmission \& transformation field, power distribution field and information communication field. In this paper, the AI image processing technology is applied in patrol inspection for surface environment of transmission channel, so as to greatly improve the surface environment analysis efficiency for transmission channel, and thus bring a high-efficient and reliable technical approach for operation, maintenance, inspection and repair of electrical grid.
\end{abstract}

Keywords: AI; image processing; feature extraction; transmission channel; surface environment.

\section{Introduction}

As a traditional industry in the national economy of our country, the power system has been playing an irreplaceable role. With the recent development of AI technology, how to integrate the traditional electrical grid technologies with the emerging computer AI technology, image processing technology and big data technologies and apply them in patrol inspection business for electrical grid so as to develop the electric grid into a more safe and reliable "intelligent electrical grid" has become the important development direction and main trend of electrical grid technologies. The surface environment monitoring for transmission channel is a major part of patrol inspection business for electric grid. Since most of transmission line equipment is located in rural environment where the meteorological conditions are complicated and the surface conditions are variable, the conductors, lightning lines, insulators and hardware may get broken, rust or overheated after long-time operation under the action of various factors. In the past, the patrol inspection was carried out by workers tower by tower, the huge workload of patrol inspection and the complicated geographical environment have brought unknown safety risks to patrol inspection personnel, and the miscellaneous and toilsome acceptance inspection and survey on iron towers and the under-developed manual patrol inspection mode have consumed a lot of human and other resources, so that the more intensified work could not be effectively unfolded; furthermore, the subjectivity of manual inspection would lead to the problems such as inconsistent inspection standards, wrong inspection results or improper record, which may, if not found in time, cause various troubles. Therefore, the large-scale surface environment monitoring for transmission lines is of great significance.

In light of the present status of patrol inspection business as mentioned above, the means such as unmanned aerial vehicle and remote sensing satellite have been used for carrying out large-scale patrol inspection, but the data obtained through picture taking and remote sensing need the secondary manual

\footnotetext{
+ Corresponding author. Tel.: +8618611580926

E-mail address: owhnet@163.com.
} 
processing, and the data processing steps are relatively complicated, so that the efficiency of patrol inspection work could not be really improved. Therefore, in this paper, an AI-based image processing technology is brought forth for quick processing and feature extraction for data acquired, which can automatically divide the four types of surface features (bare land, buildings, vegetation and roads), and intelligently monitor and analyze the change in surface features, so as to provide a new solution for the development of patrol inspection technologies for electrical grid, greatly save the human resources, and improve the efficiency and accuracy of surface environment inspection for transmission channel.

\section{AI-based Image Processing Technologies}

\subsection{Digital image processing methods}

The main process of digital image processing is divided into the following three steps: input image preprocessing, image feature extraction and image feature analysis [1]. The input image preprocessing plays an important role in image analysis process, and is the foundation for study on image information. Since the cameras may be affected by various external factors in the course of acquisition of image, the uncertainty and complication of images and the illumination will affect the subsequent feature extraction and analysis [2]. Therefore, the image preprocessing technology is used to enhance the useful information of image, and weaken other unuseful redundant information. The common image preliminary processing technologies include grey level transformation, size normalization and histogram equalization for image.

The grey level transformation means to transform a colored image into a grey level image while the location of every pixel point in image remains unchanged. As for ordinary colored images, the RGB model is used to decide the value of every pixel. However, the use of grey level image can improve the effect of original image and increase the contrast of original image, so as to make the subsequent calculation more accurate. The image size normalization means to use a series of geometrical size adjustment to ensure that the original images have the unified size or location feature. In the course of image feature extraction, training or classification, a lot of images may be used, and the size normalization for image can ensure that the images processed have the same geometric feature, so as to ensure that the subsequent feature extraction or training can be smoothly carried out [3]. The image size normalization mainly includes scaling, translation and rotation. The histogram of an image indicates the statistical relationship between every grey level and the times that such grey level appears, and is an important means to express the image feature. If the pixel of an image is evenly distributed in all possible grey levels, then its image has the relatively high contrast and variable grey level tune, and the grey levels shown are richer. The histogram equalization means to change the unevenly-distributed histogram of an original image into an evenly-distributed histogram. Through histogram equalization, an image will have the improved contrast, which is helpful for subsequent analysis and processing. In this paper, the image with RGB3 wavebands gets its grey levels changed in every waveband, and then goes through the subsequent processing..

\subsection{Feature extraction and analysis for digital images}

The feature extraction for digital image is the most important step in image recognition process, and is the precondition for subsequent accurate analysis and classification. The common feature extraction methods include principal component analysis method, LBP operator method and wavelet analysis method [4]. The principal component analysis method is a method based on unsupervised learning. Its main thought is to transform through linear transformation the original feature data into a group of lower-dimension feature data which retain the main feature components of original data and ignore other components which are not meaningful for subsequent classification. The local binary pattern (LBP) is mainly used to describe the texture feature of image, and its main thought is to compare the neighborhood pixel with central pixel so as to obtain the code 0 or 1 for such neighborhood pixel, and then clockwise connect the neighborhood pixels in parallel so as to obtain the LBP code of central pixel [5]-[7]. Its disadvantage is that it can only describe the information in one dimension rather than in several dimensions and directions. The Gabor wavelet method is a classic local feature extraction method, and can realize the better description of change in texture. The Gabor wavelet filter is developed on the basis of Fourier transformation, and it absorbs the Gauss window function on the basis of Fourier transformation. Through adjustment of parameters of window function, the 
kernel functions in different dimensions and directions can be obtained; by using such kernel functions, the vectors in feature area can be extracted. Normally, the image information which has gone through the Gabor wavelet transformation has higher dimension, so that it needs to go through the dimension-lowering process.

The feature analysis for digital image is based on the previous feature extraction, and the feature classification is often carried out in this step. The common classification methods mainly include artificial neural network (ANN) method, support vector machine method and k-nearest neighbor (KNN) method. The artificial neural network simulates the neuron structure in human brain, learns the relationship between input and output through continuous training, is a frequently-used method in the field of machine learning, and is also frequently used in face recognition and numerical recognition. The support vector machine was brought forth by Corinna Cortes and Vapnik in 1995, and is a kind of model classifier based on supervised learning. Its main thought is to transform the input space into higher-dimension space through nonlinear transformation and finally solve the optimal linear interface. The support vector machine is also a frequentlyused classifier in the field of machine learning. The k-nearest neighbor method is also widely used in pattern recognition field. Its main thought is that, if $\mathrm{k}$ similar samples of a tested image belong to a same class, then the tested image also belongs to such class.

\section{AI-based Inspection For Change In Surface Environment Of Transmission Channel}

In this paper, an AI-based inspection method for change in surface environment of transmission channel is realized on the basis of FCN algorithm, and the flowchart of such algorithm is indicated in Figure 1.

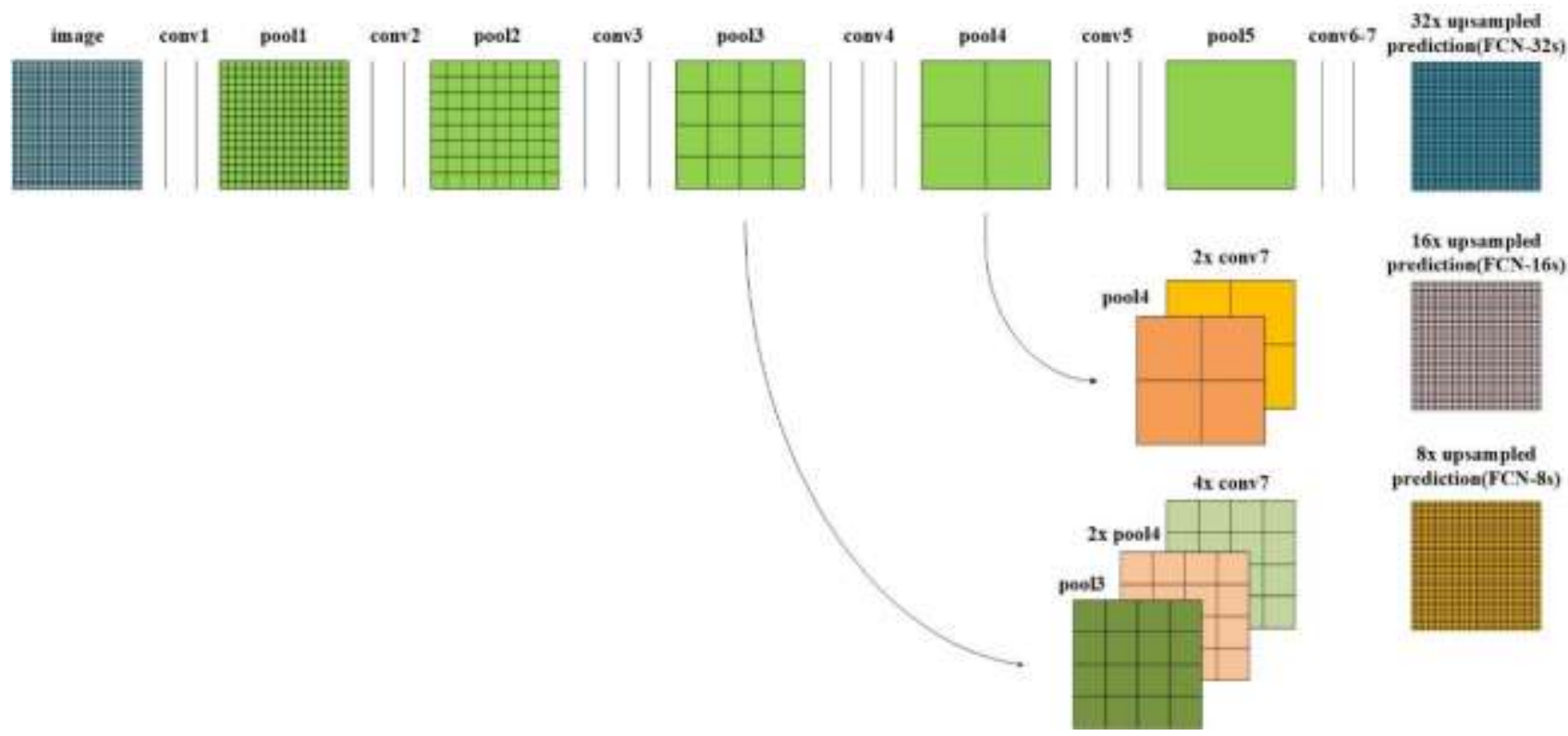

Fig 1: Structural Diagram of FCN-8s

Firstly, the GF-2 image is divided and adjusted into appropriate size, so as to get adapted to network structure. In this paper, in order to accelerate the extraction by FCN of type of land feature, the KNN is firstly used to sort out the divided images, and the images with cloud or shaded area are weeded so as to reduce the time spent by FCN in processing of non-land-feature images. In the FCN, there are five $2 \times 2$ down-sampling layers, and after going through every down-sampling layer, the image will get its resolution reduced. As a result, the resolution of feature diagram from the last convolutional layer will be very low, and the detail feature will be not obvious. If the 32-time up-sampling is directly carried out for feature diagram, the image precision of image obtained will be not high, and the edge will be relatively rough. Jonathan et al. proposed the FCN structure wherein the multi-dimensional features are integrated. This structure, in conjunction with the feature diagrams obtained from different down-sampling layers, ensures that the model has higher detail-processing ability. FCN has 5down-sampling layer, there are several convolutional layers in front of every down-sampling layer, and the image from the last layer is heat map. FCN has three different feature integration methods. The method whereby the heat map directly goes through 32-time up-sampling is 
called as FCN-32s; the method whereby the heat map goes through 2-time up-sampling, is integrated with the feature diagram from the 4thlayer down-sampling layer and then goes through 16-time up-sampling is called as FCN-16s; the method whereby the feature diagram obtained by using FCN-16s goes through 2-time up-sampling, is integrated with the feature diagram with the 3rd layer down-sampling layer and then goes through 8-time up-sampling is called as FCN-8s [8], [9]. Figure 1 is the schematic diagram of FCN -8s. In this paper, the $\mathrm{FCN}-8 \mathrm{~s}$ is used for training and subsequent prediction of data.

When the model is being predicted, the RGB, three convolutional channels will be respectively used to carry out featuring sampling for RGB, grey level diagram and edge examination diagram, and will send out three heat maps at their ends. These three heat maps will go through weighted superimposition, be combined into a heat map, and then be sent to the deconvolutional channel. Thereafter, the feature integration method FCN-8s will be used to integrate the heat map and down-sampling diagram, and then a resulting diagram will be sent out through up-sampling.

In this paper, the cross entropy function softmax is taken as the loss function of model, and its mathematical expression is indicated in Formula (1):

$$
C=-\sum_{i} y_{i} \ln a_{i},\left(a_{i}=e^{z_{i}} / \sum_{k} e^{z_{k}}, Z_{i}=\sum_{j} w_{i j} X_{i j}+b\right)
$$

Wherein: represents the jth weight of the ith neuron; is the offset; represents the ith output of such network; represents the output value of softmax, namely the predicted value corresponding to every pixel point; represents the true classification result, namely the label value of every pixel point; the Dropout is added in the course of training, so as to randomly deactivate some concealed layer nodes whenever the model is being trained. This method can effectively reduce the number of parameters selected in every training, and prevent the occurrence of overfitting phenomenon.

\section{Application and Result}

This experiment adopts the Tensorflow machine learning framework on Windows and uses the Anaconda software to carry out programming, and the configuration of experiment platform is as follows: the CPU is Inter Xeon E5- 2650 3.5GHz, and the GPU is NVIDIA Tesla K80.

\subsection{Data source}

In the classification algorithm for deep learning, the quality of training set directly decides the quality of trained model. The training set is divided into original images (including RGB, Gary and feature images) and the corresponding label images. The label images are made on the basis of the manually-allocated vector files, and the RGB value of specific label is indicated in the table. In order to save the internal memory and get adapted to the network structure, the trained images are cut into $256 * 256$ images for training. In this experiment, a total of 10,000 pairs of original images and label images are selected, including 8,000 pairs in training set and 2,000 pairs in validation set, and there is no overlap between training set and validation set. Figure 2 indicates the data of training dataset, and Figure 3 indicates the label dataset corresponding to the training dataset.

Table 1: RGB Value of Various Land Features

\begin{tabular}{cccc}
\hline Name & Red & Green & Blue \\
\hline Bare land & 128 & 255 & 128 \\
Building & 0 & 0 & 128 \\
Vegetation & 0 & 166 & 0 \\
Road & 255 & 255 & 0 \\
Shadow & 0 & 0 & 0 \\
\hline
\end{tabular}



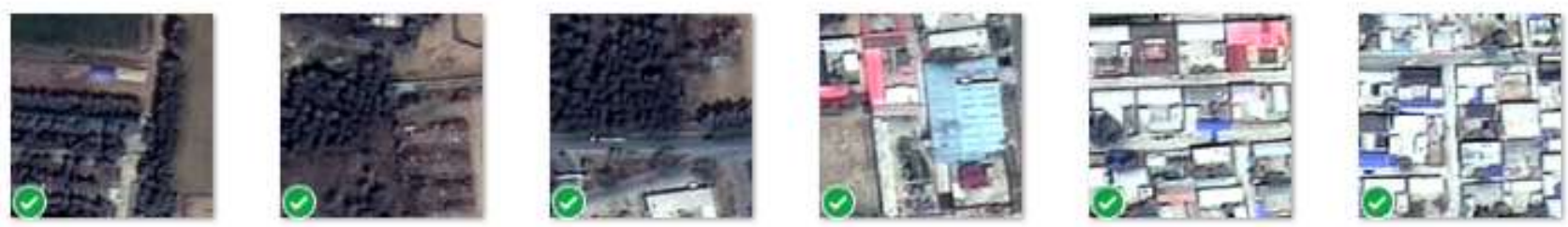

Fig 2: Training Dataset
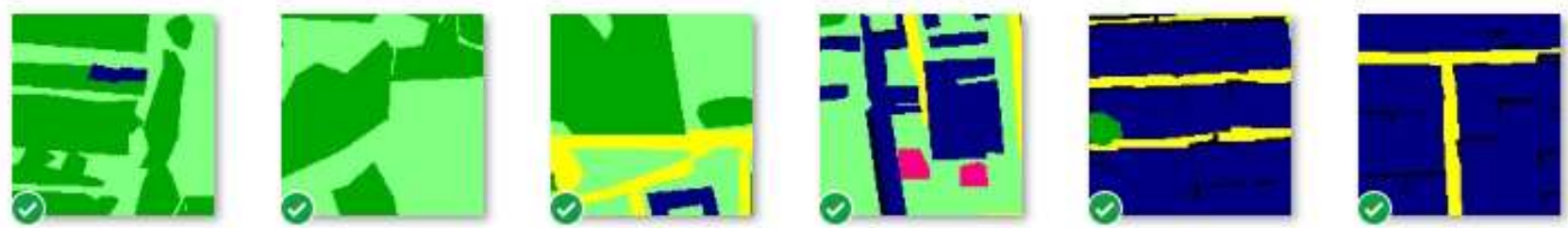

Fig 3: Label Dataset Corresponding to Training Dataset

\subsection{Application examples}

In this paper, on the basis of the above-mentioned data sets, the training parameters for Tensor flow are set as follows: the Batch-size is 64 , the Epoachs is 162 , and the learning_rate is 0.0001 . Figure 4 is the postiteration loss curve.

training_loss

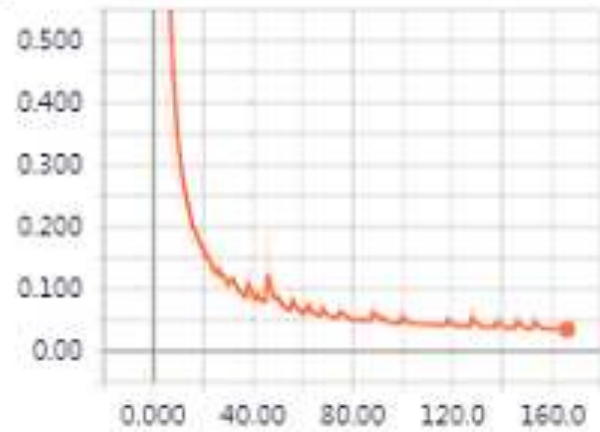

validation_loss

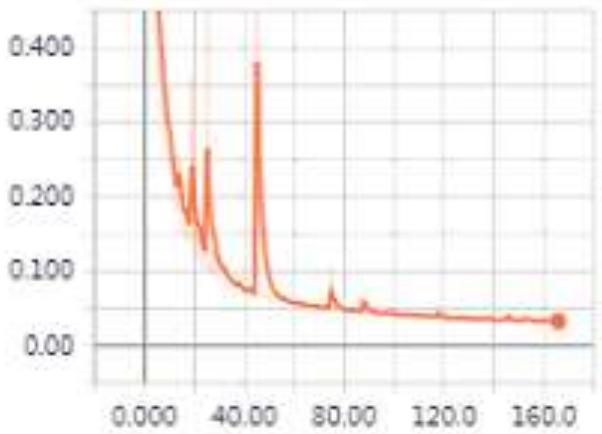

Fig 4: Loss Curve of Tensor Board
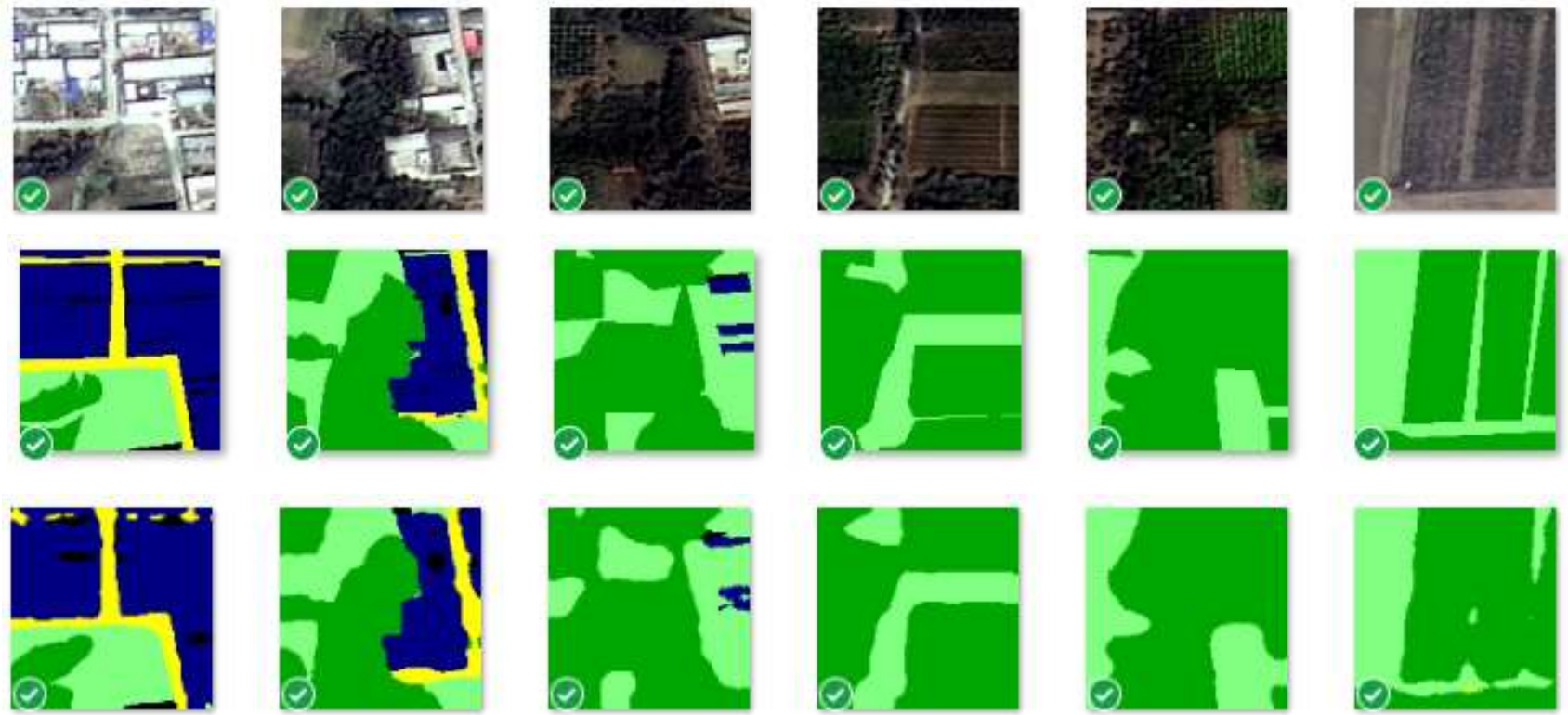

Fig 5: Predict result (Top: Origin Dataset; Middle: Ground Truth; Bottom: Predicted Dataset) 
The time spent for calculation of predicted data set is less than 2 minutes. Figure 5 is the schematic diagram of final predicted data set. With respect to precision, the comparison result between data in prediction set and data in validation set is as follows: the pixel accuracy is 0.931880 , the IoU (Intersection over Union) is 0.743998 , Table 2 gives the precision indicators for various land features. The result value indicates that, by using this method, the automatic large-scale patrol inspection for surface environment of transmission channel can be realized in a relatively-precise manner. With respect to precision evaluation, the evaluation is carried out for every type of recall, precision and F1-score, and the calculation formula is indicated in Formula 2- Formula 4.

$$
\begin{array}{r}
\text { recall }=\frac{T P}{T P+F N} \\
\text { precision }=\frac{T P}{T P+F P} \\
F_{1}=2 \frac{\text { precision } \times \text { recall }}{\text { precision }+ \text { recall }}
\end{array}
$$

Table 2: Precision Indicators of Various Land Features

\begin{tabular}{cccc}
\hline Label Name & Precision & Recall & F1 score \\
\hline Bare land & 0.900794 & 0.938656 & 0.919335 \\
Building & 0.955662 & 0.944691 & 0.950145 \\
Vegetation & 0.966436 & 0.944709 & 0.955449 \\
Road & 0.888688 & 0.820249 & 0.853098 \\
Shadow & 0.398008 & 0.568695 & 0.468283 \\
\hline
\end{tabular}

\section{Conclusion}

The power system is the important pillar for normal operation of society, and the safety and stability of power transmission provides the important guaranty for the living of the public and the development of economy. With the development of new-generation computer technologies and the continuous improvement of scale of electrical grid, the intelligent electrical grid which integrates the advanced AI technologies and image processing technologies has become the main transformation direction of traditional electrical grid, and the traditional manual operation has been gradually replaced by highly-intelligent technical means, which greatly improves the efficiency and also ensures the safety and stability of system. In this paper, the application scenarios and technical route for an AI-based technology for patrol inspection for surface environment of electrical grid are brought forth. In the experimental environment, the model training and prediction are carried out for a lot of samples through image preprocessing and feature extraction and analysis, so as to save a lot of labor costs and time, ensure the precision of interpretation of surface environment to a certain extent, and thus develop the power patrol inspection in the intelligent and informatized direction.

\section{Acknowledgements}

This work was supported by a grant from National Key R\&D Program of China (2018YFC0809400), the science \& technology project of State Grid (Research and application of special geological structure detection for transmission line corridor based on small radar, GCB17201700142), and the Academy Innovation Fund of China Electric Power Institution (5242001700FA).

\section{References}

[1] Sampedro C, Martinez C, Chauhan A, et al. A supervised approach to electric tower detection and classification for power line inspection[C]. International Joint Conference on Neural Networks. IEEE, 2014:1970-1977.

[2] Yu P, Dong B G, Xue Y J. Electric Power Tower Inclination Angle Detection Method Based on SIFT Feature Matching[J]. Applied Mechanics \& Materials, 2012, 236-237:759-764. 
[3] Zhang W, Huang H, Schmitz M, et al. Effective Fusion of Multi-Modal Remote Sensing Data in a Fully Convolutional Network for Semantic Labeling[J]. Remote Sensing, 2017, 10(1):52.

[4] Golightly I, Jones D. Corner detection and matching for visual tracking during power line inspection[J]. Image \& Vision Computing, 2003, 21(9):827-840.

[5] Lin Qingda, Xuan Liang, Tan Weiwei et al. Summary of Study on Image Recognition for Power System[J]. Yunnan Electric Power, 2017, 45(4):30-33.

[6] Wen Shihua. Application of Computer Science in Intelligent Electrical Grid [J]. China Hi-tech Enterprises, 2016(21):47-49.

[7] Li Hongwei. Intelligent Visual System Applied in Intelligent Transformer Substation[J]. Electric Power Automation Equipment, 2012, 32(8):141-147.

[8] Long J, Shelhamer E, Darrell T. Fully convolutional networks for semantic segmentation[C]. IEEE Conference on Computer Vision and Pattern Recognition. IEEE Computer Society, 2015:3431-3440.

[9] Yu H, Yang Z, Tan L, et al. Methods and Datasets on Semantic Segmentation: A review[J]. Neuro computing, 2018. 